\title{
XXX. On a machine for calculating the products, quotients, logarithms, and powers of numbers
}

\section{Rev. Henry Moseley M.A. F.R.S.}

To cite this article: Rev. Henry Moseley M.A. F.R.S. (1847) XXX. On a machine for calculating the products, quotients, logarithms, and powers of numbers, Philosophical Magazine Series 3, 30:200, 171-179, DOI: $10.1080 / 14786444708645633$

To link to this article: http://dx.doi.org/10.1080/14786444708645633

曲 Published online: 30 Apr 2009.

Submit your article to this journal $\lceil\pi$

Џ Article views: 3

Q View related articles $\square$ 
rise to crystallization, like those of frost and of congealed water. Although we have rarely seen it in a liquid state, yet during summer, under the influence of the sun's rays, its crystals become perfectly liquid and assume a globular form, and which they retain as they again solidify; forming, in these cases, conditions corresponding to water in a liquid state, ice and sleet.

XXX. On a Machine for Calculating the Products, Quatients, Logarithms, and Powers of Numbers. By the Rev. Henry Moseley, M.A., F.R.S., one of Her Majesty's Inspectors of Schools, and lately Professor of Natural Philosophy and Astronomy in King's College, London*.

[With a Plate.]

T HAVE proposed to myself in the construction of this machine, to determine mechanically the products, quotients, logarithms, squares, square routs, and other powers and roots of the natural numbers, by means of combinations of greater simplicity than have hitherto been applied to the purposes of mechanical calculation.

The accompanying Plate II. is intended to illustrate the principle of the machine, but it does not show the mechanical details of its construction or the due proportion of its parts,

It will be observed that it consists principally of a cone and a screw. The application of the cone and the disc to various purposes of mechanical calculation has long been well known, and particularly by the ingenious applications made of the disc to dynamical admeasurement by MM. Poncelet and Morin.

The novelty of this instrument consists in the combination of the screw with the cone.

CD represents a small screw terminated by solid cylindrical axes or gudgeons, of which that shown by the letter $D$ is pro= longed, and carries an index $T$. The screw adinits of being fixed in its bearings or made to revolve in them.

$P Q$ is a wheel having a hollow cylindrical axis, into which is accurately fitted a hollow cylindrical piece, whose internal surface is traversed by the thread of a female screw working upon the male screw $\mathrm{CD}$.

The hollow axis of the wheel PQ admits of being fixed upon the cylindrical piece inserted in it, or of being disengaged from that piece, so that the wheel $P Q$ may be made to carry the female screw round with it in its revolutions, or to turn freely upon the outside of the cylinder which contains that screw, as upon an axis.

- Communicated by the Author.

$\mathrm{N} 2$ 
Phil Mag.S.3. VoL XXX Plate. II

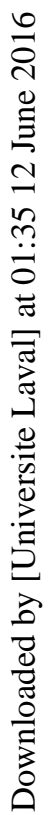
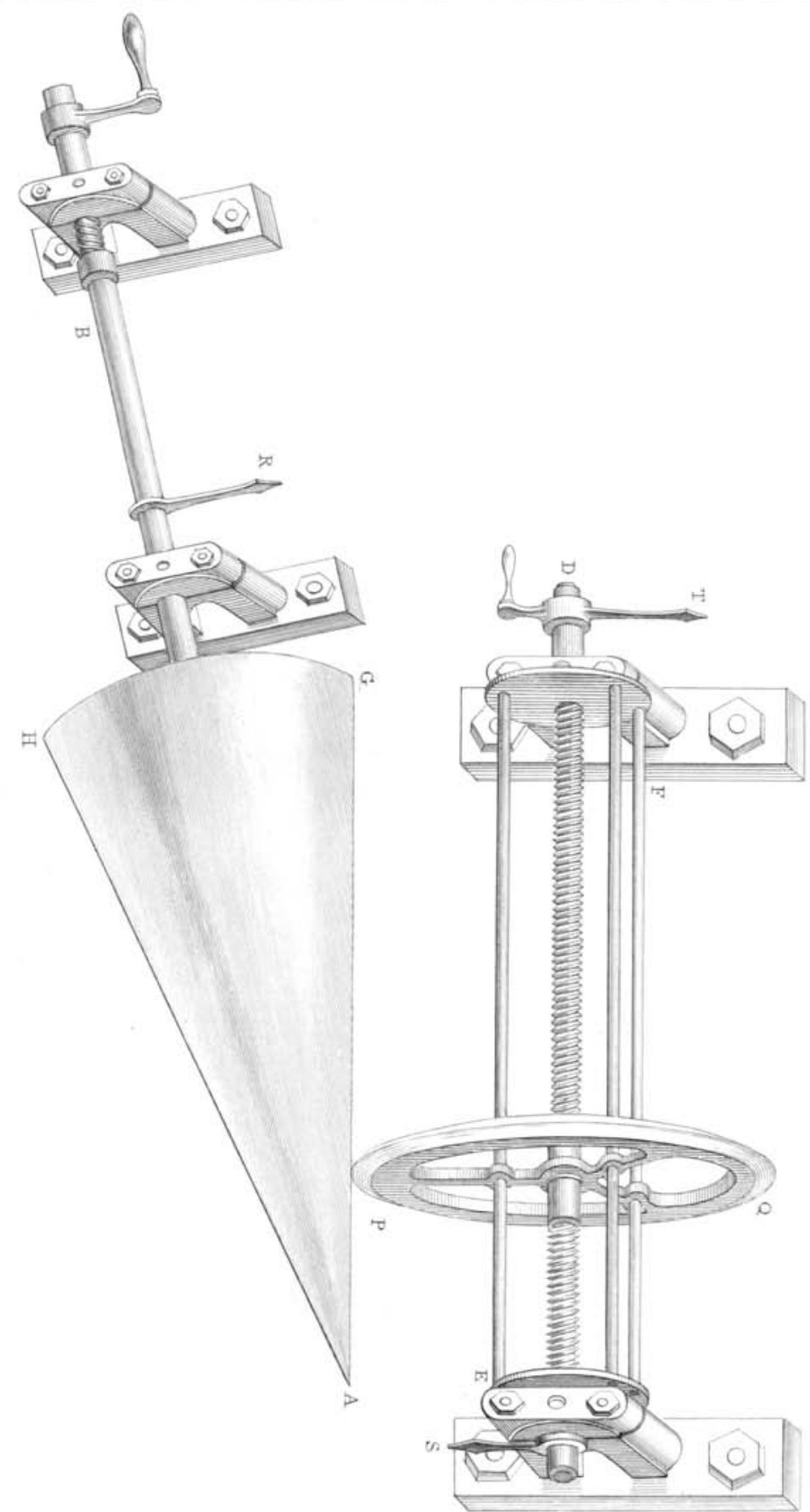

( 


\section{Rev. H. Moseley on a Machine for Calculating the}

The three arms of the wheel PQ are pierced by apertures, through which pass, accurately fitting them, the three rods of the rigid frame $\mathrm{EF}$, which frame turns in fixed bearings at $\mathrm{C}$ and $\mathrm{D}$ upon hollow axes, through which axes pass the cylindrical extremities or gudgeons of the screw CD.

The wheel $P Q$ traverses the frame EF longitudinally, and at the same time carries it round with it in its revolutions : and it admits of being fixed upon the frame at any distance from either extremity. The frame carries an index $\mathrm{S}$.

AGH is a cone, whose side AG is parallel to the screw $C D$, which turns by means of a spindle $A B$ upon fixed bearings, and which is continually pressed upon the edge of the wheel PQ by a spring acting upon the extremity of its spindle. The cone is of some soft metal, brass for instance. The edge of the wheel $P Q$ is very thin, of steel, and milled, so that it imprints a minute tooth upon the soft face of the cone at every point where it revolves in contact with it.

The spindle $A B$ carries an index $R$. The indices $R, S, T$ point to equidistant divisions upon fixed circles, not shown in the Plate; and each of them is connected with a train of wheels and pinions, whose numbers of teeth are in the proportion of ten to one, and which serve to measure the complete number of revolutions and parts of a revolution made by each index.

Now certain dimensions (hereafter to be determined) being assigned to the different elements of this combination, it may be shown,-

1st. That if the fiame EF be fixed, and the screw CD turned uncil the index $T$ points to any given number $N_{3}$, and then the frame be released, the wheel PQ fixed upon the frame, and the cone turned until the index $R$ points to any other given number $\mathrm{N}_{1}$, then will the index $S$ point to the product of the numbers $\mathrm{N}_{1}$ and $\mathrm{N}_{3}$.

2nd. That if, as before, the frame EF be fixed, and the screw turned until the index $T$ points to any number $\mathrm{N}_{3}$, and then the wheel $P Q$ be fixed on the frame, the frame released, and the cone turned until the index $S$ points to any other number $\mathrm{N}_{2}$, then will the index $R$ point to the quotient of $\mathrm{N}_{2}$ by $\mathrm{N}_{3}$.

3rd. That if the screrw $C D$ be fixed, the wheel $P Q$ and the frame released, and certain other adjustments made, and if the cone be turned until the index $S$ points to any number $N$, then will the index $\mathrm{R}$ point to the logarithm of that number.

4th. That if the spindle of the cone be connected with the axis of the screw CD by means of two bevelled wheels, so that the rotation of the one shall always bear a given relation to that of the other, then the frame EF and the wheel PQ being 
both released, and the cone turned until the index $R$ points to any number, the index $S$ will point to the square of that number.

\section{Theory of the Instrument.}

Let be taken to represent half the angle at the apex of the cone, $\rho$ the radius of the wheel $P Q$, and $\lambda$ the distance between each two threads of the screw; also let $n_{1}, n_{2}, n_{3}$ represent severally the numbers of equidistant divisions on the circles to which the indices $R, S, T$ severally point; then will $\frac{N_{1}}{n_{1}}, \frac{N_{2}}{n_{2}}, \frac{N_{3}}{n_{3}}$ represent the numbers of revolutions made severally by the indices whilst the numbers $N_{1}, N_{2}, N_{3}$ are registered. Now suppose that when the wheel $P Q$ is at the apex of the cone the indices $R, S$ and $T$ each point to zero, and the frame EF being fixed, let the screw CD be turned until the index $\mathrm{T}$ shows the number $\mathrm{N}_{3}$; then will the screw have made $\frac{N_{3}}{n_{3}}$ revolutions, and the wheel $P Q$ will have advanced from the apex of the cone to a distance $A P$ from it, represented by $\frac{N_{3}}{n_{3}} \cdot \lambda$; so that the radius $\mathrm{PM}$ of the circle described by that point whilst the cone revolves will be represented by $\frac{N_{3}}{n_{3}} \lambda \sin$, and its circumference by $2 \pi \frac{N_{3}}{n_{3}} \lambda \sin$.. Now let the cone be made to revolve (the wheel PQ being fixed upon the frame and the frame released) until the index $R$ shows the number $N_{1}$, or until the cone has made $\frac{N_{1}}{n_{1}}$ revolutions; then will the point $P$ in it have been made to revolve through a space represented by

$$
2 \pi \frac{\mathrm{N}_{3}}{n_{3}} \cdot \lambda \sin \iota \cdot \frac{\mathrm{N}_{3}}{n_{1}},
$$

or by

$$
\frac{2 \pi \lambda \sin 6}{n_{1} n_{3}} \cdot \mathrm{N}_{1} \mathrm{~N}_{3} \text {. }
$$

But by reason of the continual pressure of the cone upon the edge of the wheel $P Q$, that wheel will have been carried round in the revolution of the cone, so that the circumference of the wheel will have described precisely the same space as the point $\mathbf{P}$ of the surface of the cone; if therefore $N_{2}$ represent the number pointed to by the index $S$, so that $\frac{N_{2}}{n_{2}}$ represents the number of revolutions made by the frame $E F$ and wheel 
174. Rev. H. Moseley on a Machine for Calculating the

$P Q$, and therefore $2 \pi p \frac{\mathrm{N}_{2}}{n_{2}}$ the space described by its circumference, we shall have

or

$$
\begin{aligned}
& 2 \pi \rho \frac{\mathrm{N}_{2}}{n_{2}}=\frac{2 \pi \lambda \sin \iota}{n_{1} n_{3}} \cdot \mathrm{N}_{1} \cdot \mathrm{N}_{3} ; \\
& \mathrm{N}_{2}=\left(\frac{n_{2} \lambda \sin \iota}{\rho n_{1} n_{3}}\right) \mathrm{N}_{1} \cdot \mathrm{N}_{3} .
\end{aligned}
$$

Let now the quantities $n_{1}, n_{2}, n_{3}, \rho, \lambda$, , be so assumed that

$$
\left(\frac{n_{2} \lambda \sin \iota}{\rho n_{1} n_{3}}\right)=1 ; \cdot \text {. . . . . }\left(\alpha_{i}\right)
$$

then

$$
\mathrm{N}_{2}=\mathrm{N}_{1} \cdot \mathrm{N}_{3} \cdot \text {. . . . . . . }
$$

that is to say, the number $\mathrm{N}_{2}$ shown by the index $\mathrm{S}$ is the product of the numbers $N_{1}$ and $N_{3}$ shown by the indices $R$ and $\mathrm{T}$.

Dividing equation (1.) by $\mathrm{N}_{3}$

$$
\mathrm{N}_{1}=\frac{\mathrm{N}_{2}}{\mathrm{~N}_{3}} ; . \cdot \cdot \cdot \cdot \cdot \cdot \text {. }
$$

whence it follows that if, proceeding in other respects precisely as before, we cause the cone to revolve, not until its own index $\mathbf{R}$ points to a given number, but until the index $S$ points to the given number $N_{2}$, then will the index $R$ of the cone show a number $\mathrm{N}_{1}$, which is the quotient of $\mathrm{N}_{2}$ and $\mathrm{N}_{3}$.

To adjust the instrument to show the logarithms of numbers, let the wheel $P Q$ be brought to the apex of the cone, and let the screw $C D$ be fixed, and the index $S$ then made to point to zero. Let the cone then be turned until the index $\mathrm{S}$ points to unity, and in this position of the cone let the index $\mathrm{R}$ be made to point to zero. The frame EF being then allowed to turn freely, and the wheel $P Q$ to traverse freely on the frame, the number $N_{1}$ shown by the index $R$ will always be the logarithm of the number $N_{2}$ shown by the index $S$. For while the number $\mathrm{N}_{1}$ is increased by the exceedingly stnall number $\Delta \mathrm{N}_{1}$, let the number $\mathrm{N}_{2}$ be increased by $\Delta \mathrm{N}_{2}$; the parts of a revolution marle by the cone and wheel respectively whilst $N_{1}$ and $N_{2}$ receive these small increments, will then be $\frac{\Delta N_{1}}{n_{1}}$ and $\frac{\Delta N_{2}}{n_{2}}$, and the arcs to radius unity, $2 \pi \frac{\Delta N_{1}}{n_{1}}$ and $2 \pi \frac{\Delta N_{3}}{n_{Q^{*}}}$. Moreover, the wheel $\mathrm{PQ}$ having made $\frac{\mathrm{N}_{2}}{n_{\mathrm{Q}_{2}}}$ revolutions upon the fixed screw $\mathrm{CD}$, will have been carried from the apex of the cone to a distance from it represented by 
$\frac{\mathrm{N}_{2}}{n_{2}} \lambda$; so that the radius PM of the circle in the act of being described by the point $P$ upon the surface of the cone whilst the numbers $\Delta \mathrm{N}_{1}$ and $\Delta \mathrm{N}_{2}$ are being registered, will be $\frac{N_{2}}{n_{2}} \lambda \sin 1 ;$ and the exceedingly small arc described by that point whilst these numbers are registered, will be represented by

$$
2 \pi \frac{\Delta \mathrm{N}_{1}}{n_{1}} \cdot \frac{\mathrm{N}_{2}}{n_{2}} \lambda \sin
$$

But this arc, described by $\mathrm{P}$ upon the cone, is equal to that described in the same time by a point in the circumference of the wheel, which last is represented by $2 \pi \frac{\Delta N_{2}}{n_{2}} \rho$;

$$
\begin{gathered}
\therefore \quad 2 \pi \frac{\Delta \mathrm{N}_{1}}{n_{1}} \cdot \frac{\mathrm{N}_{2}}{n_{2}} \cdot \lambda \sin \iota=2 \pi \frac{\Delta \mathrm{N}_{2}}{n_{2}} \rho ; \\
\therefore\left(\frac{\lambda \sin \iota}{n_{1} \rho}\right) \Delta \mathrm{N}_{1}=\frac{\Delta \mathrm{N}_{2}}{\mathrm{~N}_{2}} ;
\end{gathered}
$$

or passing to the limit and integrating between the limits unity and $\mathrm{N}_{2}$,

$$
\begin{aligned}
& \left(\frac{\lambda \sin \iota}{n_{1} \rho}\right) \mathrm{N}_{1}=\log _{\varepsilon} \mathrm{N}_{2} ; \\
& \therefore\left(\frac{\lambda \sin \iota}{\varepsilon_{t} \rho}\right)^{\mathrm{N}_{\mathrm{t}}}=\mathrm{N}_{2^{*}}
\end{aligned}
$$

Let the quantities $\lambda, 1, n_{1}, \rho$ be so assumed that

$$
\begin{aligned}
& \frac{\lambda \sin t}{n_{\mathrm{I} \varphi}}=10 \text {; . . . . . . . } \\
& \therefore 10 \mathrm{~N}_{\mathrm{r}}=\mathrm{N}_{2} \text {; } \\
& \therefore \mathrm{N}_{1}=\log _{10} \mathrm{~N}_{2} \text {. }
\end{aligned}
$$

whence it follows that the number shown by the index $R$ (under this adjustment of the instrument) will in every position be the common logarithm of the number then shown by $S$.

If the spindle $\mathrm{AB}$ be made to carry round with it the screw $C D$ by the intervention of two bevel wheels the numbers of whose teeth bear a given ratio, $n$, to one another, so that the screw $C D$ may make $n$ revolutions or parts of a revolution whilst the spindle AB makes one; if, moreover, the wheel PQ be so released from the female screw, which forms its centre, as that the outer surface of that screw may serve for an axis about which it may turn freely whilst it is still carried along the frame by the longitudinal motion of the screw; and if the in- 
dices be all made to point to zero when the wheel $P Q$ is at the apex of the cone, then, representing as before by $\mathrm{N}_{1}, \mathrm{~N}_{2}$, $\mathrm{N}_{3}$ the numbers shown at any time by the indices, $n \frac{\mathrm{N}_{1}}{n_{1}}$ will represent the number of revolutions made by the screw whilst the number $\mathrm{N}_{1}$ is registered by $\mathrm{R}$, and therefore $\frac{n \mathrm{~N}_{1}}{n_{1}} \lambda$ the distance AP by which the wheel $P Q$ will have been moved from the vertex of the cone; so that $\frac{n N_{1}}{n_{1}} \lambda$ sin $\downarrow$ will be the radius $P M$ of the circle which the point $P$ of the cone is in the act of describing, and $\frac{n \mathrm{~N}_{1}}{n_{1}} \lambda \sin 1.2 \pi \frac{\Delta \mathrm{N}_{1}}{n_{1}}$ the small arc which it actually describes whilst the increment $\Delta N_{1}$ is registered by the index $R$. But $\Delta N_{2}$ being at the same time registered by the index $S$, the circumference of the wheel will describe a space represented by $\frac{\Delta \mathrm{N}_{2}}{n_{2}} \cdot 2 \pi \rho$;

$$
\begin{aligned}
& \therefore \frac{\Delta \mathrm{N}_{2}}{n_{2}} \cdot 2 \pi \rho=\frac{n \mathrm{~N}_{1}}{n_{1}} \lambda \sin \iota \cdot 2 \pi \frac{\Delta \mathrm{N}_{1}}{n_{1}} ; \\
& \therefore \Delta \mathrm{N}_{2}=\left(\frac{\lambda n n_{2} \sin \iota}{n_{1}^{2} \rho}\right) \cdot \mathrm{N}_{1} \Delta \mathrm{N}_{1} .
\end{aligned}
$$

And passing to the limit and integrating between the limits zero and $\mathrm{N}_{1}$,

$$
\mathrm{N}_{2}=\left(\frac{\lambda n n_{2} \sin \iota}{2 n_{1}^{2} \rho}\right) \cdot \mathrm{N}_{1}^{2}
$$

If, therefore, the quantities $\lambda, n, n_{1}, n_{2}, \rho$, be so assumed that

$$
\left(\frac{\lambda n n_{2} \sin \iota}{2 n_{1}^{2} \rho}\right)=1, . . \cdot \cdot \cdot(\gamma \cdot)
$$

then

$$
\mathrm{N}_{2}=\mathrm{N}_{1}^{2} \text {, . . . . . . }
$$

and

$$
\mathrm{N}_{1}=\sqrt{\mathrm{N}_{2}} ;
$$

so that the number $\mathrm{N}_{2}$ shown by the index $\mathrm{S}$ (under this adjustment of the instrument) is, in every position, the square of the number shown by the index $R$; and conversely, the number shown by the index $R$ is, in every position, the square root of the number shown by $S$.

If a second frame and screw and wheel, similar to $\mathrm{EF}$ and $\mathrm{CD}$ and $\mathrm{PQ}$, be conceived to be placed parallel to the side AH of the cone, and if the cone be so pressed upon the edges of both these wheels as to carry them round with it in its revolution; if, moreover, the axis of the second screw be so connected 
with the axis of the frame EF by two bevel wheels, as that the number of revolutions made by the latter shall bear a certain given ratio $n^{\prime}$ to the number made in the same time by the former; then will the number $\mathrm{N}_{4}$ shown at any time by the index of this last frame be the cube of the number $\mathrm{N}_{1}$ shown at the same time by the index $R$.

For $\frac{\mathrm{N}_{2}}{n_{2}}$ representing the number of revolutions made by the first frame $\mathrm{EF}$ whilst the number $\mathrm{N}_{1}$ is registered, $n^{\prime} \frac{\mathrm{N}_{2}}{n_{2}}$ will represent the number of revolutions made in the same time by the second screw, so that $n^{\prime} \frac{N_{2}}{n_{2}} \cdot \lambda$ will represent the distance by which the second wheel will have been moved from the apex of the cone, and $n^{\prime} \frac{\mathrm{N}_{2}}{n_{2}} \lambda$ sin, the radius of the circle which its point of contact with the cone will be in the act of describing. Moreover, $2 \pi \frac{\Delta N_{1}}{n_{1}}$ is the angle which the cone describes whilst $\Delta N_{1}$ is registered by the index $R$; so that $n^{\prime} \frac{\mathrm{N}_{2}}{n_{2}} \lambda \sin 1.2 \pi \frac{\Delta \mathrm{N}_{1}}{n_{1}}$ is the small arc described during this time by the point of contact of the second wheel with the cone. But the increment $\Delta N_{4}$ being registered during that time by the index of the second frame, the circumference of the second wheel will describe the arc $\frac{\Delta N_{4}}{n_{4}} \cdot 2 \pi \rho\left(n_{4}\right.$ representing the number of equal parts into which the circle is divided to which the index of the second frame points);

but $\mathbf{N}_{2}=\mathrm{N}_{1}^{2}$ (by equation 4 );

$$
\begin{aligned}
\therefore n^{\prime} & \frac{\mathrm{N}_{2}}{n_{2}} \lambda \sin 1.2 \pi \frac{\Delta \mathrm{N}_{1}}{n_{1}}=\frac{\Delta \mathrm{N}_{4}}{n_{4}} \cdot 2 \pi \rho ; \\
\therefore & \Delta \mathrm{N}_{4}=\left(\frac{n^{\prime} n_{4} \lambda \sin }{n_{1} n_{2} \rho}\right) \cdot \mathrm{N}_{2} \cdot \Delta \mathrm{N}_{1} ;
\end{aligned}
$$

$$
\therefore \Delta \mathrm{N}_{4}=\left(\frac{n^{\prime} n_{4} \lambda \sin \iota}{n_{1} n_{2} \rho}\right) \cdot \mathrm{N}_{1}^{2} \cdot \Delta \mathrm{N}_{1} \text {. }
$$

Therefore, passing to the limit and integrating,

If therefore $n^{\prime}$ be so taken that

$$
\mathrm{N}_{4}=\left(\frac{n^{\prime} n_{4} \lambda \sin \imath}{3 n_{1} n_{\rho}}\right) \cdot \mathrm{N}_{1}{ }^{3} \text {. }
$$

$$
\frac{n^{\prime} n_{4} \lambda \sin \iota}{3 n_{1} n_{2 P}}=1, \ldots . . . . . . .
$$


178 Rev. H. Moseley on a Machine for Calculating Numbers.

then

$$
\begin{aligned}
& \mathrm{N}_{4}=\mathrm{N}_{1}^{8}, . . . . \\
& \mathrm{N}_{1}=\sqrt[3]{\mathrm{N}_{4}} ; \text {. . . . . . . }
\end{aligned}
$$

so that (under this combination) the number $\mathrm{N}_{4}$ shown by the index of the second frame is, in every position, the cule of that shown by the index $R$; and conver'sely, the number shown by the index $\mathbf{R}$ is the cube root of that shown, at the same time, by the index of the second frame.

In like manner, by placing any number of like systems of a frame, screw and wheel, upon the side of the cone, the third screw receiving its motion from the second frame, the fourth from the third, \&c., any other powers of the number $\mathrm{N}_{1}$, or roots of the numbers $\mathrm{N}_{2}, \mathrm{~N}_{4}$, \& c. may be conceived to be determined, the power shown by the last index, or the root by the first, being one greater than the number of frames so applied.

The four equations of condition $\left(\alpha_{.}\right),\left(\beta_{*}\right),(\gamma),.\left(\delta_{\text {. }}\right)$ between the nine quantities $n_{1}, n_{2}, n_{3}, n_{4}, n, n^{\prime}, \lambda$, $1, \rho$ leave five of them undetermined. These are to be assumed of such values as may be found most convenient in the construction of the machine, the general principle of which it is the object of this paper to explain.

The Plate in particular, as has already been stated, is by no means intended to show the details of the construction of the machine, or even the general proportions of its parts, but simply to illustrate its principle. There are, indeed, some elements of its construction, which, for the sake of simplicity, have been altogether omitted from it. These are the trains of wheels which must be connected with each of the axes carrying the indices $\mathrm{R}, \mathrm{S}, \mathrm{T}$ to register the complete numbers of revolutions made by those indices, and the divided circles to which the indices point, showing such portions of the numbers $\mathrm{N}_{1}, \mathrm{~N}_{4}$, \&c. as correspond to less than one complete revolution.

It will be obvious that, since the number $\mathrm{N}_{2}$ represents (in the first adjustment of the instrument) the product of the numbers $N_{1}$ and $N_{3}$, the divisions on the circles pointed to by the indices $R$ and $T$ must be greater than those of the circle pointed to by the index $\mathrm{S}$, or the numbers $n_{1}$ and $n_{3}$ less than the number $n_{2^{*}}$.

It will, moreover, be observed, that the instrument, whilst it differs from other calculating machines, and claims to be superior to them in the simplicity of its combinations, differs also, and is inferior to them in this respect, that the truth of its registrations is dependent on the mechanical accuracy of its construction.

Those elements of the machine, on the mechanical truth 
and adaptation of which its accuracy depends, are,-1st, the screw, the convolutions of whose thread must be of geometrical uniformity; 2nd, the cone, whose surface must be of geometrical truth; and 3rd, the wheel, whose edge must have so intimate a contact with, and hold upon, the surface of the cone, as to partake accurately in its motion at every point which it traverses.

My experience in the use of a similar cone and wheel in a steam indicator constructed at the expense of the British Association of Science, has convinced me that the required accuracy is in this last respect attainable.

In respect to the two first-mentioned sources of error, it may be observed that there are no mechanical forms of greater simplicity than the cone and the screw, and probably none in respect to which greater truth of construction is attainable.

Every such instrument must however have its error; its amount in respect to this instrument may be determined by an obvious method, and it is probable that it may in every case be corrected by a corresponding adjustment.

XXXI. On the use of a mixture of Spirit of Wine and Camphine, as a Light for Optical Purposes. By John Gronge Children, Esq., F.R.S.

\section{To Richard Taylor, Esq.}

My dear Sir,

Halstead Place, Feb. 13, 1847.

$F$ you think the following trifle worth inserting in the Phi1 losophical Magazine, it is at your service. I have lately assisted a friend in getting up an apparatus for dissolving views, \&c. on a scale too large for sufficient illumination by the best Argand lamp, and we considered condensed hydrogen gas as too dangerous an agent for a plaything; especially in private houses in the country, where much must be left in the preparation of the gases, \&c. to servants unused to such duties. We set to work therefore to find a substitute that might answer our purpose; and after a few trials we obtained a tolerable light, by throwing the flame of spirit of wine on a surface of quicklime by a current of oxygen gas, something after the manner of Lieut. Drummond's original experiment*. The illumination by the flame of spirit of wine alone, however, proved to be too feeble; but by mixing a portion of camphine (spirit of turpentine) with it (which readily dissolves in alcohol)

* Philosophical Transnctions, 1826, p. 330. Drummond's object and ours were somewhat different. He wanted to get an intense but small sphere of light, "adapted to the nature of a (parabolic) reflector:" we also wanted as intense a light as we could obtain, but, as we could not advantageously use a reflector, one which should likewise extend over a much larger surface. 